International Journal of Advanced Astronomy, $9(1)(2021) 32-37$
International Journal of Advanced Astronomy
SPC
Website: www.sciencepubco.com/index.php/IJAA
Research paper

\title{
Periodic orbit in the photo-gravitational restricted three body problem around the collinear Lagrangian points when more massive primary is an oblate spheroid and source of radiation
}

\author{
Derick John ${ }^{1}$ *, Ram Krishan Sharma ${ }^{2}$ \\ ${ }^{1}$ Department of Aerospace Engineering, Karunya Institute of Technology and Sciences, Coimbatore - 641114, India \\ ${ }^{2}$ Department of Aerospace Engineering, Karunya Institute of Technology and Sciences, Coimbatore - 641114, India \\ *Corresponding author E-mail: derickcjohn@gmail.com
}

\begin{abstract}
The circular Restricted Three Body Problem is considered with the more massive primary as an oblate spheroid and source of radiation. A new mean motion expression given by $\mathrm{n} 2=1+6 \mathrm{~A}$ is used in the present study, when the secular effect of the oblateness on the mean motion, argument of perigee and right ascension of the ascending node is considered. The locations of the collinear Lagrangian points are found. It is found to have some variation from the previous study conducted on the same because of the new mean motion that is considered in this study. The variations of the location of the Lagrangian points due to the unperturbed as well as the perturbed problem due to oblateness and radiation pressure are studied. A study on the eccentricity e and angular frequency s at L1, L2 and L3 is carried out. It is observed how the change in effect of oblateness and radiation pressure has affected the location, angular frequency and eccentricity at L3, though there are only small changes noticed in the case of L1 and L2.
\end{abstract}

Keywords: Angular Frequency; Collinear Lagrangian Points; Oblateness; Periodic Orbits; Radiation Pressure; Restricted Three Body Problem.

\section{Introduction}

The restricted three-body problem describes the motion of an infinitesimal mass moving under the gravitational effect of the two finite masses, called primaries, which move in circular orbits around their center of mass on account of their mutual attraction and the infinitesimal mass not influencing the motion of the primaries. The RTBP (restricted three-body problem) possesses five stationary solutions called Lagrangian points, three of which are called collinear equilibria, lie on the line joining the primaries and the other two called equilateral equilibria make equilateral triangles with primaries. In general, the collinear equilibria are unstable while equilateral points are stable only in a certain region for the mass parameter. The shapes of the participating bodies in the RTBP are assumed to be oblate. The oblateness of a body can produce perturbation deviation from the two-body motion. In the RTBP it is assumed that the infinitesimal mass moves under the mutual gravitational force of the primaries. In RTBP when at least one of the interacting bodies is an intense emitter of radiation the repulsive force of the radiation pressure is considered. In the RTBP the origin is considered to be the center of mass of the two finite mass bodies and take axes rotating with the masses, such that they lie along the $\mathrm{x}$-axis. In the dimensionless system of variables $\mu_{1}$ and $\mu_{2}$ represents the masses of the primaries and the sum of two masses is therefore unity and whose distance are also unity. In this study the larger mass is taken on the right side. The book of Szebehely (1967) provides systematic coverage of the literature on the subject as well as derivations of some of the important results.

Various authors have made studies on Lagrangian points in the restricted three-body problem by considering the more massive primary or both primaries as source of radiation. Some of the important contributions are by Radzievsky (1950, 1953), Chernikov (1970). Some of the significant studies carried out related to the Lagrangian points by considering the oblateness of one or both the primaries with their equatorial planes coincident with the plane of motion, are by Vidyakin (1974), Sharma (1975), Subba Rao \& Sharma (1975), Sharma \& Subba Rao $(1975,1976,1978)$.

In this paper we study the RTBP when the more massive primary is a source of radiation as well as an oblate spheroid. A study on the same had been done by (Namboodiri et al. 2008) but with the mean motion value of $1+3 \mathrm{~A} / 2$. In this paper when the secular effect of the oblateness on the mean motion, argument of perigee and right ascension of the ascending node is considered the mean motion is taken to be 1+6A derived analytically by (Sharma et al. 2020). We have derived the expressions for locations of the collinear points and a numerical study on the effect of oblateness and radiation pressure is carried out. We have also carried out study on the effect of oblateness and radiation pressure on the angular frequency and eccentricity with new mean motion. 


\section{Equation of motion}

In the dimensionless synodic coordinate system (x, y), the equations of motion are (Szebehely 1967)

$\ddot{x}-2 n \dot{y}=\frac{\partial \Omega}{\partial x}$,

$\ddot{\mathrm{y}}+2 \mathrm{n} \dot{\mathrm{x}}=\frac{\partial \Omega}{\partial \mathrm{y}}$

Where

$\Omega=\frac{n^{2}}{2}\left[(1-\mu) r_{1}^{2}+\mu r_{2}^{2}\right]+\frac{q(1-\mu)}{r_{1}}+\frac{\mu}{r_{2}}+\frac{q(1-\mu) A}{2 r_{1}^{3}}$

With $r_{1}^{2}=(x-\mu)^{2}+y^{2}, r_{2}^{2}=(x+1-\mu)^{2}+y^{2}, n^{2}=1+6 A$.

The mass parameter $\mu=m_{1} /\left(m_{1}+m_{2}\right)$, where $m_{1}$ and $m_{2}$ are the masses of the primaries $m_{1}>m_{2}$, such that $m_{1}+m_{2}=1$ the oblateness coefficient $A=\frac{A E^{2}-A P^{2}}{5 R^{2}}$, where $A E$ and $A P$ are the dimensional equatorial and polar radii of the more massive primary and $\mathrm{R}$ is the distance between the primaries. The term $\mathrm{q}$ is due to radiation pressure. If $\mathrm{q}=1$, then there is no effect of radiation pressure. For equilibrium points $\Omega_{\mathrm{x}}=\Omega_{\mathrm{y}}=0$

Therefore

$$
\begin{aligned}
& \mathrm{n}^{2} \mathrm{x}-\frac{\mathrm{q}(1-\mu)(\mathrm{x}-\mu)}{\mathrm{r}_{1}^{3}}-\frac{\mu(\mathrm{x}-\mu+1)}{\mathrm{r}_{2}^{3}}-\frac{3 \mathrm{Aq}(1-\mu)(\mathrm{x}-\mu)}{2 \mathrm{r}_{1}^{5}}=0, \\
& \mathrm{y}\left[\mathrm{n}^{2}-\frac{\mathrm{q}(1-\mathrm{u})}{\mathrm{r}_{1}^{3}}-\frac{\mathrm{u}}{\mathrm{r}_{2}^{3}}-\frac{3 A q(1-u)}{2 \mathrm{r}_{1}^{5}}\right]=0
\end{aligned}
$$

\section{Location of collinear equilibrium points}

When $\mathrm{y}=0$, eq. (

collinear points $\mathrm{L}_{1}\left(\mathrm{x}_{1}, 0\right), \mathrm{L}_{2}\left(\mathrm{x}_{2}, 0\right), \mathrm{L}_{3}\left(\mathrm{x}_{3}, 0\right)$, where

(3) determines the locations of the

$\mathrm{x}_{1}=\mu-1-\xi_{1}, \mathrm{x}_{2}=\mu-1+\xi_{2}, \mathrm{x}_{3}=\mu+\xi_{3}$.

$\xi_{1}, \xi_{2}, \xi_{3}$ satisfies the seventh-degree polynomials

$$
\begin{array}{r}
(12 A+2) \xi_{1}^{7}+[(-12 A-2) \mu+60 A+10] \xi_{1}^{6}+[(-48 A-8) \mu+120 A+20] \xi_{1}^{5}+[(2 q-72 A-14) \mu-2 q+120 A+20] \xi_{1}^{4}+ \\
{[(4 q-48 A-16) \mu-4 q+60 A+10] \xi_{1}^{3}+[[(3 A+2) q-12 A-14] \mu+(-3 A-2) q+12 A+2] \xi_{1}^{2}-8 \mu \xi_{1}-2 \mu=0}
\end{array}
$$

$$
\begin{aligned}
& (12 A+2) \xi_{2}^{7}+[(12 A+2) \mu-60 A-10] \xi_{2}^{6}+[(-48 A-8) \mu+120 A+20] \xi_{2}^{5}+[(-2 q+72 A+10) \mu+2 q-120 A-20] \xi_{2}^{4}+ \\
& {[(4 q-48 A) \mu-4 q+60 A+10] \xi_{2}^{3}+[[(-3 A-2) q+12 A-10] \mu+(3 A+2) q-12 A-2] \xi_{2}^{2}+8 \mu \xi_{2}-2 \mu=0,} \\
& (12 A+2) \xi_{3}^{7}+[(12 A+2) \mu+24 A+4] \xi_{3}^{6}+[(24 A+4) \mu+12 A+2] \xi_{3}^{5}+[(2 q+12 A) \mu-2 q] \xi_{3}^{4}+(4 q \mu-4 q) \xi_{3}^{3}+[(3 A+2) q \mu+ \\
& (-3 A-2) q] \xi_{3}^{2}+(6 A q \mu-6 A q) \xi_{3}+3 A q \mu-3 A q=0 .
\end{aligned}
$$

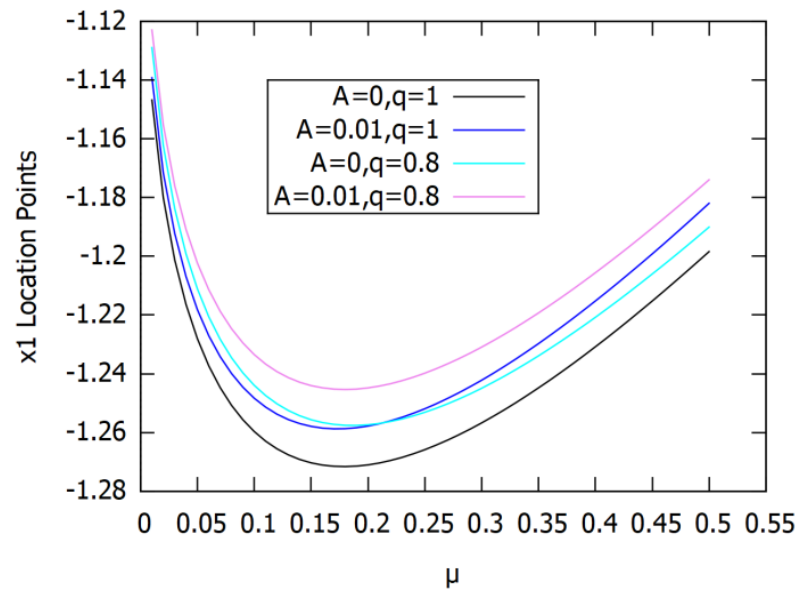

Fig. 1: Graph Showing the Variation of Location of L1 versus $\mu$. 


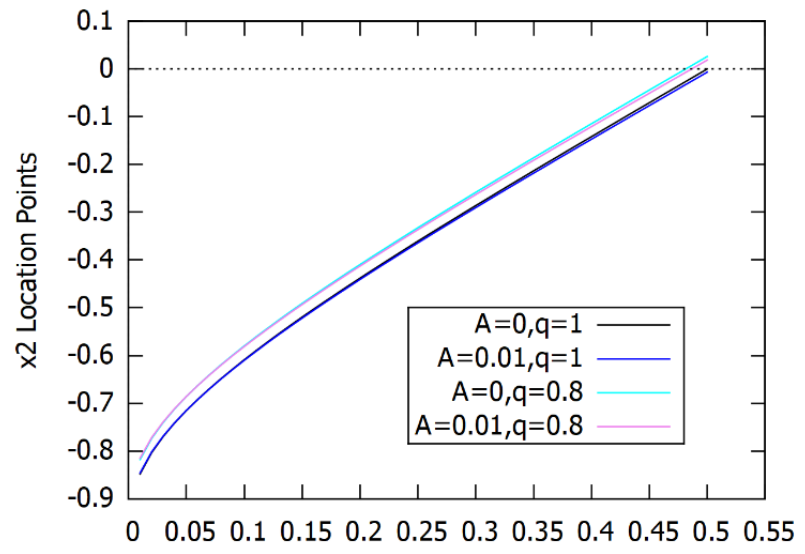

Fig. 2: Graph Showing the Variation of Location of L2 Versus $\mu$.

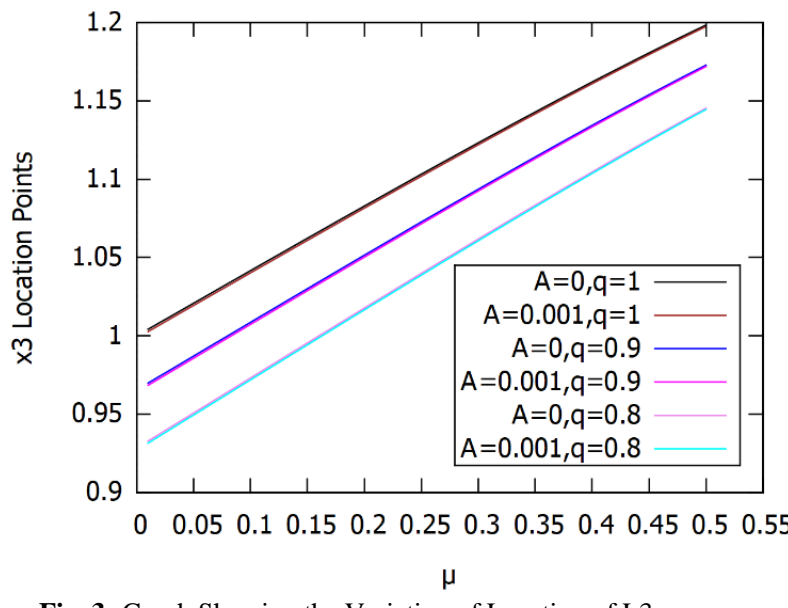

Fig. 3: Graph Showing the Variation of Location of L3 versus $\mu$.

Fig, 1, 2 and 3 provides the locations of the collinear points $L_{1}, L_{2}$ and $L_{3}$ for $\mu$ from 0 to 0.5 for different values of $A_{1}$ and $q$. In Fig. 1 it is noted that the collinear point $\mathrm{L}_{1}$ comes nearer to the primaries with the increase in oblateness and radiation pressure, and also it is noted that at smaller values of mass parameter $\mu$, the location moves away from the primaries, but moves towards the primaries for the values of $\mu$ above 0.2. In Fig. 2 it is seen that $\mathrm{L}_{2}$ move away from the more massive primary with the increase in oblateness and come nearer to it with the increase in radiation pressure. Fig. 3 shows that $\mathrm{L}_{3}$ moves closer to the primaries with the increase in oblateness and radiation pressure.

\section{Variational equations and characteristic exponents}

The variational equations in the linear analysis becomes

$\xi^{\prime \prime}-2 n \eta^{\prime}=\Omega_{\mathrm{xx}}(\mathrm{a}, \mathrm{b}) \xi+\Omega_{\mathrm{xy}}(\mathrm{a}, \mathrm{b}) \eta$,

$\eta^{\prime \prime}+2 \mathrm{n} \xi^{\prime}=\Omega_{\mathrm{xy}}(\mathrm{a}, \mathrm{b}) \xi+\Omega_{\mathrm{yy}}(\mathrm{a}, \mathrm{b}) \eta$.

The characteristic equation of (9) is given by

$\lambda^{4}+\left(4 \mathrm{n}^{2}-\Omega_{\mathrm{xx}}-\Omega_{\mathrm{yy}}\right) \lambda^{2}+\Omega_{\mathrm{xx}} \Omega_{\mathrm{yy}}-\Omega_{\mathrm{xy}}{ }^{2}=0$.

At the collinear points, we get

$\Omega_{\mathrm{xx}}=\mathrm{n}^{2}+\frac{2 \mathrm{q}(1-\mu)}{\mathrm{r}_{1}^{3}}+\frac{2 \mu}{\mathrm{r}_{2}^{3}}+\frac{6 \mathrm{q}(1-\mu) \mathrm{A}}{\mathrm{r}_{1}^{5}}>0$,

$\Omega_{\mathrm{xy}}=0$,

$\Omega_{\mathrm{yy}}=\mathrm{n}^{2}-\frac{\mathrm{q}(1-\mu)}{\mathrm{r}_{1}^{3}}-\frac{\mu}{\mathrm{r}_{2}^{3}}-\frac{3 \mathrm{q}(1-\mu) \mathrm{A}}{2 \mathrm{r}_{1}^{5}}<0$.

Consequently, $\Omega_{\mathrm{xx}} \Omega_{\mathrm{yy}}-\Omega_{\mathrm{xy}}^{2}<0$.

We can note that the roots $\lambda_{\mathrm{i}}(\mathrm{i}=1,2,3,4)$ of the characteristic equation (10) are 
$\lambda_{1,2}= \pm\left[-\beta_{1}+\left(\beta_{1}^{2}+\beta_{2}^{2}\right)^{\frac{1}{2}}\right]^{\frac{1}{2}}= \pm \lambda$,

$\lambda_{3,4}= \pm\left[-\beta_{1}-\left(\beta_{1}^{2}+\beta_{2}^{2}\right)^{\frac{1}{2}}\right]^{\frac{1}{2}}= \pm \mathrm{is}$

Where

$\beta_{1}=2-\frac{\Omega_{\mathrm{xx}}+\Omega_{\mathrm{yy}}}{2}$,

$\beta_{2}^{2}=-\Omega_{\mathrm{xx}} \Omega_{\mathrm{yy}}>0$.

It is noted that $\lambda_{1,2}$ are real and $\lambda_{3,4}$ are pure imaginary. Hence, in general case the collinear equilibria are unstable. The eccentricity of the periodic orbit is given by $\mathrm{e}=\sqrt{1-\beta_{3}^{-2}}$.

Where

$\beta_{3}=\left(s^{2}+\Omega_{x x}\right) / 2 n s$

The angular frequency $s$ is given by $s=\sqrt{\beta_{1}+\sqrt{\beta_{1}^{2}+\beta_{2}^{2}}}$.

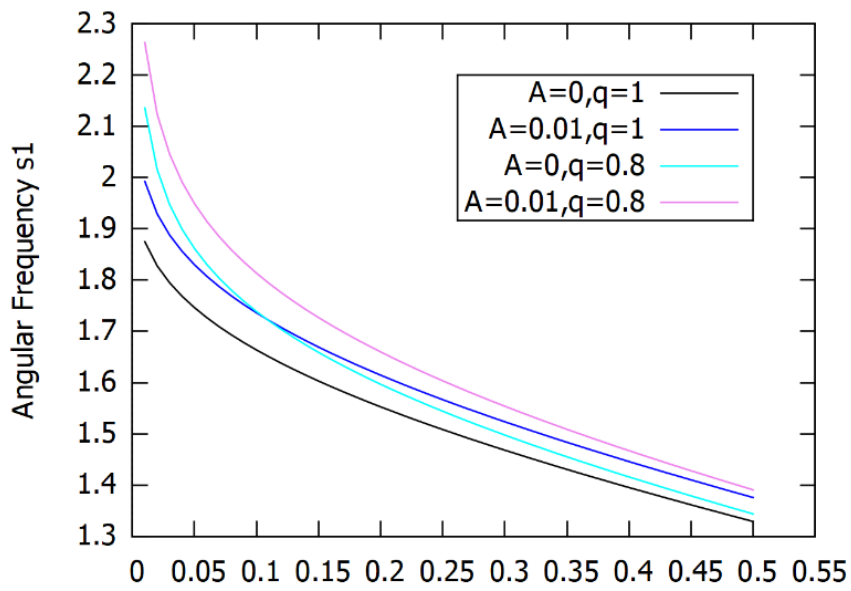

Fig. 4: Graph Showing the Variation of Angular Frequency at L1

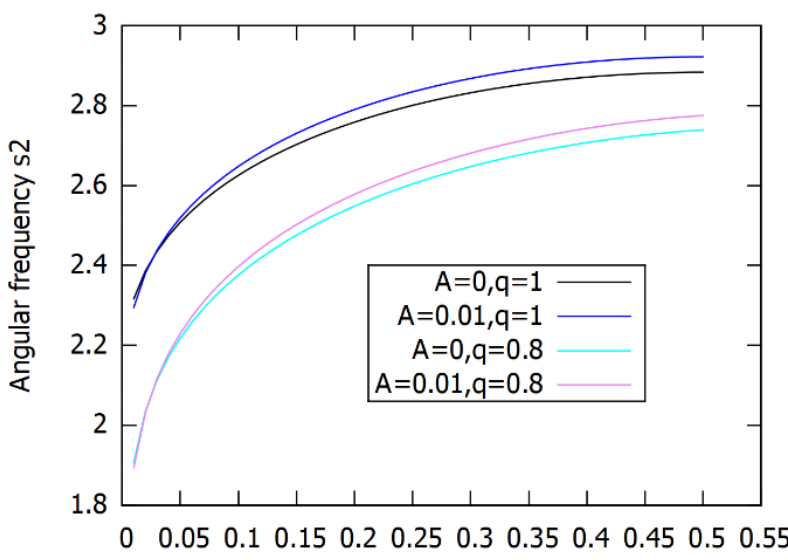

Fig. 5: Graph Showing the Variation of Angular Frequency at L2. 


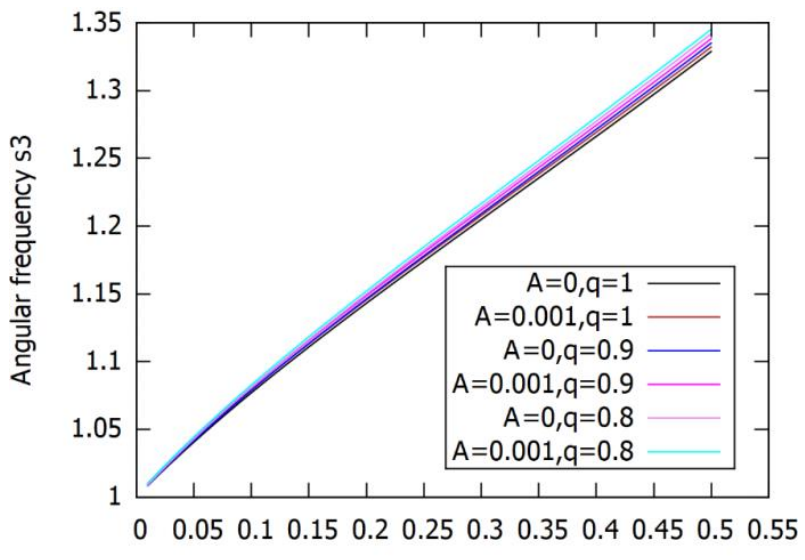

Fig. 6: Graph Showing the Variation of Angular Frequency at L3

Fig. 4, 5 and 6 provide the angular frequencies $s_{1}, s_{2}$ and $s_{3}$ at $L_{1}, L_{2}$ and $L_{3}$, respectively for the mass parameter $\mu$ up to 0.5 for different values of $\mathrm{A}_{1}$ and q. It may be noted from Fig. 4 that the increase in the effect of oblateness and radiation pressure increases $\mathrm{s}_{1}$ values for $\mathrm{L}_{1}$. At lower values of $\mu$ the difference between the angular frequencies for radiation pressure is high but at higher values of $\mu$ the difference is less. From Fig. 5 it may be noted that the angular frequency $\mathrm{s}_{2}$ at $\mathrm{L}_{2}$ increases with oblateness and decreases with increase in radiation pressure q. At lower values of $\mu$ the increase in $s_{2}$ is noticeable but as the $\mu$ value increases the variation in $s_{2}$ decreases. In Fig. 6 it is seen that, at $\mathrm{L}_{3}$ the increase in oblateness and radiation pressure increases $\mathrm{s} 3$.

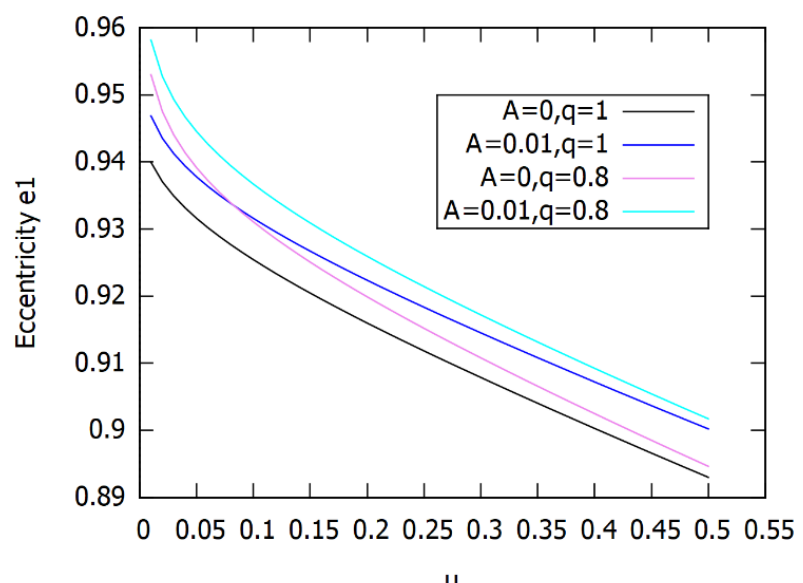

Fig. 7: Graph Showing the Variation of Eccentricity at L1

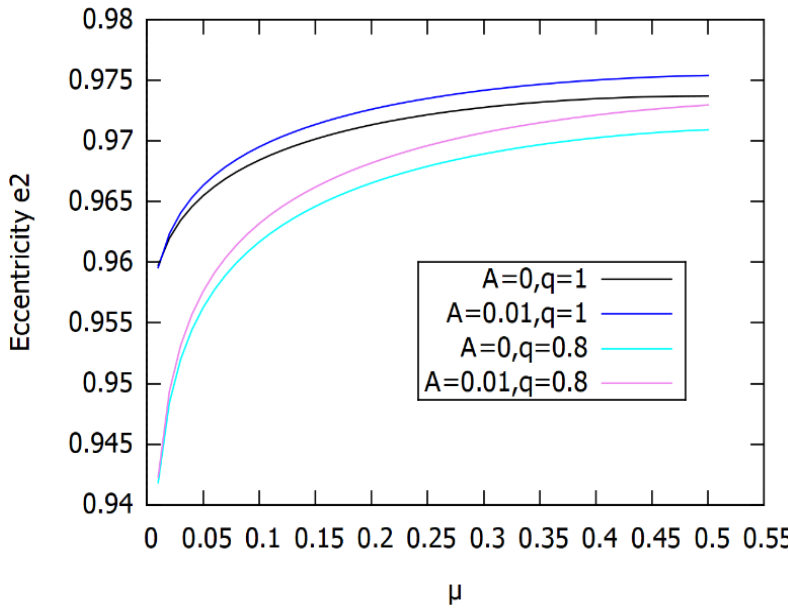

Fig. 8: Graph Showing the Variation of Eccentricity at L2. 


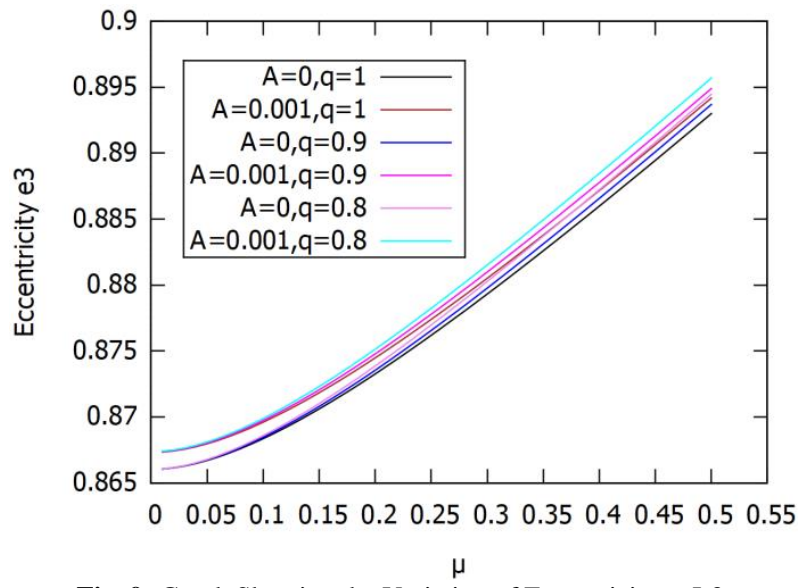

Fig. 9: Graph Showing the Variation of Eccentricity at L3.

Fig. 7, 8 and 9 provide the eccentricity $\mathrm{e}_{1}, \mathrm{e}_{2}$ and $\mathrm{e}_{3}$ at $\mathrm{L}_{1}, \mathrm{~L}_{2}$ and $\mathrm{L}_{3}$ for the mass parameter $\mu$ up to 0.5 for different values of $\mathrm{A}_{1}$ and q. It may be noted from Fig. 7 that at $\mathrm{L}_{1}$ the increase in oblateness and radiation pressure increases eccentricity, also as $\mu$ increases the eccentricity decreases. It is seen from Fig. 8 that for $\mathrm{L}_{2}$ eccentricity increases with oblateness and decreases with increase in radiation pressure, also at $\mu<0.1$ the increase in eccentricity is very high but becomes almost constant for values of $\mu$ above 0.25 . From Fig. 9 at $\mathrm{L}_{3}$ the eccentricity increases with oblateness and radiation pressure and the increase become linear at $\mu>0.15$.

\section{Conclusion}

A study on the effect of oblateness and radiation pressure on the location, angular frequency and the eccentricity of the conditional periodic orbits at $\mathrm{L}_{1}, \mathrm{~L}_{2}$ and $\mathrm{L}_{3}$ is carried out with the new mean motion $\mathrm{n}^{2}=1+6 \mathrm{~A}$. The present study is different from the previous study conducted on the same with mean motion $n^{2}=1+3 \mathrm{~A} / 2$ by (Namboodiri et al. 2008). In the current study the mean motion includes the secular effect of the oblateness on the mean motion, argument of perigee and right ascension of the ascending node. The effect of this new mean motion can be seen when the two studies are compared. It can be noticed how the change in effect of oblateness and radiation pressure has affected the location, angular frequency and eccentricity at $\mathrm{L}_{3}$, though the changes at $\mathrm{L}_{1}$ and $\mathrm{L}_{2}$ due to the effects of oblateness and radiation is barely noticeable.

\section{References}

[1] Arohan, R., Sharma, R. K. (2020) Periodic orbits in the planar restricted photo-gravitational problem when the smaller primary is an oblate spheroid. Indian Journal of Science and Technology 13(16), 1630 - 1640. https://doi.org/10.17485/IJST/v13i16.401.

[2] Chernikov, V.A. (1970) The Photogravitational Restricted three-body problem. Astronomicheskii Zhurnal 47, 217. Available at: https://articles.adsabs.harvard.edu/pdf/1970SvA....14..176C (accessed 21 December 21).

[3] Kumar, S., Ishwar, B. (2011) Location of collinear equilibrium points in the generalised photogravitational elliptic restricted three body problem. International Journal of Engineering, Science and Technology 3(2), 157-162. https://doi.org/10.4314/ijest.v3i2.68143.

[4] Namboodiri, N. I. V, Reddy, D. S., Sharma, R. K. (2008) Effect of oblateness and radiation pressure on angular frequencies at collinear points. Astrophysics Space Science 318, 161 - 168. https://doi.org/10.1007/s10509-008-9934-0.

[5] Radzievsky, V.V. (1950) the restricted problem of three-bodies taking account of light pressure. Astronomicheskii Zhurnal 27, 250-256

[6] Radzievsky, V.V. (1953) the spatial case of the restricted problem of three radiating and gravitating bodies. Astronomicheskii Zhurnal 30, 265-273

[7] Raheem, A., A.R., Singh, J. (2006) Combined effects of perturbations, radiation, and oblateness on the stability of equilibrium points in the restricted three-body problem. The Astronomical Journal 131(3), 1880-1885. https://doi.org/10.1086/499300.

[8] Raheem, A., A.R., Singh, J. (2008) Combined effects of perturbations, radiation, and oblateness on the periodic orbits in the restricted three-body problem. Astrophysics Space Science 317, 9-13. https://doi.org/10.1007/s10509-008-9841-4.

[9] Sharma, R.K. (1975) Perturbations of Lagrangian points in the restricted three-body problem. Indian Journal of Pure and Applied Mathematics 6, $1099-1102$.

[10] Sharma, R. K. (1987) The Linear stability of libration points of the photogravitational restricted three-body problem when the smaller primary is an oblate spheroid. Astrophysics and Science 135, 271 - 281. https://doi.org/10.1007/BF00641562.

[11] Sharma, R. K., Sellamuthu, H., Jency, A. A. (2020) Perturbed Trojan dynamics in the solar system. AAS AIAA Astrodynamics Specialist Conference 20 - 704. Available at: https://www.researchgate.net/publication/343547691 (accessed 21 December 2021).

[12] Sharma, R.K., Subba Rao, P.V. (1975) collinear equilibria and their characteristics exponents in the restricted three-body problem when the primaries are oblate spheroids. Celestial Mechanics and Dynamical Astronomy 12, 189-201. https://doi.org/10.1007/BF01230211.

[13] Sharma, R.K., Subba Rao, P.V. (1976) Stationary solutions and their characteristic exponents in the restricted three-body problem when the more massive primary is an oblate spheroid. Celestial Mechanics and Dynamical Astronomy 13, 137-149. https://doi.org/10.1007/BF01232721.

[14] Sharma, R.K., Subba Rao, P.V. (1978) A case of commensurability induced by oblateness. Celestial Mechanics and Dynamical Astronomy 18, 185-194. https://doi.org/10.1007/BF01228715.

[15] Simmons, J.F.L., McDonald, A.J.C., Brown, J.C. (1985) The restricted 3-body problem with radiation pressure. Celestial Mechanics and Dynamical Astronomy, 35 145-187. https://doi.org/10.1007/BF01227667.

[16] Singh, J., Umar, A. (2012) Motion in the photogravitational elliptic restricted three-body problem under an oblate primary. The American Astronomical Society 143(5), 109-131. https://doi.org/10.1088/0004-6256/143/5/109.

[17] Subba Rao, P.V., Sharma, R.K. (1975) A note on the stability of the triangular points of equilibrium in the restricted three-body problem. Astronomy and Astrophysics 43, 381-383. Available at: https://articles.adsabs.harvard.edu/pdf/1975A\%26A...43..381S (accessed 21 December 2021).

[18] Szebehely, V. (1967) THEORY OF ORBITS the Restricted Problem of Three Bodies. New York and London: Academic Press. https://doi.org/10.1016/B978-0-12-395732-0.50016-7.

[19] Vidyakin, V. (1974) Astronomicheskii Zhurnal 51, 1087-1094. 